\title{
GENERAL THEORY FOR THE OUTER PLANETS
}

\author{
P. BRETACiNON and G. FRANCOU \\ Bureau des Longitudes, URA 707 CNRS, \\ 77 av. Denfert-Rochereau, 75014 Paris, France
}

\begin{abstract}
An iterative method for the construction of planetary theories has been developed in order to determine the high order perturbations with respect to the masses. These perturbations are indeed needed to enlarge the validity span of analytical theories up to some million years. The application to the simplified Sun-Jupiter-Saturn problem gives a solution accurate over several ten million years. Throughout the study of the four outer planets we meet with convergence difficulties especially in the determination of fundamental frequencies. One of the results of this study is it shows evidence of long period terms with large amplitude in the mean longitudes: $12000^{\prime \prime}$ in Saturn longitude, $20000^{\prime \prime}$ in that of Uranus.
\end{abstract}

\section{Introduction}

The long period evolution of the planetary orbits can be studied either by the construction of analytical planetary theories or by the numerical integration of the equations of motion.

The numerical integration of the motion of planets has been especially developed since 1984 with the integration of the 5 outer planets over 5 million years (Kinoshita and Nakai, 1984), over 214 million years (Applegate et al, 1986) over 100 million years (Milani et al, 1987; Nobili et al, 1989). A three million year integration was recently performed for all the planets by (Quinn et al, 1991).

The most recent analytical constructions were performed at the Bureau des Longitudes or in connection with it: Bretagnon (1974 and 1984), Duriez (1979), Laskar (1988 and 1990).

Up to now general planetary theories have been developed to the first and second order with respect to the planetary masses.

The aim of this paper is to study the contribution of the perturbations of the 3rd order and of higher orders in the planetary solutions. These perturbations are especially important in the case of planets such as Jupiter, Saturn, Uranus and Neptune. They, particularly, modify the frequency $g_{6}$ by $1 \%$ (see Laskar 1988). Moreover, to the third order with respect to the masses before integration, long period terms are found in the semi major axes. By integration of the mean motion, connected to the semi major axis by the third law of Kepler, we get long period perturbations of very large amplitude in the mean longitudes.

\section{Method for the Construction of Solutions}

Just as in the case of secular variation theories (see Bretagnon, 1982) one can build up the right hand members of the Lagrange equations either by approximations order after order with respect to the planetary masses, or by an iterative method.

In the methods of construction order after order with respect to the masses, we have to determine the second derivatives of the Lagrange equations with respect to all the elements in order to get the 3rd order perturbations, the third derivatives for the 4th order perturbations ... This brings great complexity in the computations. 
Besides it is necessary to reach a very high order, since the third order perturbations modify the frequency $g_{6}\left(=28^{\prime \prime} .23\right.$ per year $)$ by $0^{\prime \prime} .3$, those of the 4 th order by some $0^{\prime \prime} .01$.

Therefore we preferred to determine the high order perturbations by the iterative method as described in (Bretagnon, 1990). In this method, the solutions have been developed in Fourier series where the $\Phi$ arguments are represented by a linear combination of the mean longitudes $\bar{\lambda}_{j}=\lambda_{j o}+n_{j} t$, of the $\psi_{j}$ arguments linked to the system in eccentricity and of the $\theta_{j}$ arguments linked to the system in inclination :

$$
\Phi=\sum_{j=5}^{8} r_{j} \bar{\lambda}_{j}+\sum_{j=5}^{8} l_{j} \psi_{j}+\sum_{j=5}^{8} m_{j} \theta_{j}
$$

The summation is made here for $j=5$ to 8 corresponding to the system of the four planets Jupiter, Saturn, Uranus and Neptune.

For the planet $i$, the variables $a, \lambda, k=e \cos \varpi, h=e \sin \varpi, q=\sin \frac{i}{2} \cos \Omega$ and $p=\sin \frac{i}{2} \sin \Omega$ are here developed in the form :

$$
\begin{aligned}
a_{i}= & a_{i 0}+\sum_{\Phi^{*}} A_{\Phi^{*}, i} \cos \Phi^{*}+\sum_{\Phi} A_{\Phi, i} \cos \Phi \\
\lambda_{i}= & \lambda_{i 0}+n_{i 0} t+\delta n_{i} t+\sum_{\Phi^{*}} B_{\Phi^{*}, i} \sin \Phi^{*}+\sum_{\Phi} B_{\Phi, i} \sin \Phi \\
k_{i}= & \sum_{k=5}^{8} \lambda_{i k} M_{k} \cos \psi_{k}+\sum_{k=5}^{8} M_{i, \psi_{k}} \cos \psi_{k}+\sum_{\Phi^{*}} \epsilon_{\Phi^{*}} M_{i, \Phi^{*}} \cos \Phi^{*} \\
& +\sum_{\Phi} \epsilon_{\Phi} M_{i, \Phi} \cos \Phi \\
h_{i}= & \sum_{k=5}^{8} \lambda_{i k} M_{k} \sin \psi_{k}+\sum_{k=5}^{8} M_{i, \psi_{k}} \sin \psi_{k}+\sum_{\Phi^{*}} M_{i, \Phi^{*}} \sin \Phi^{*} \\
& +\sum_{\Phi} M_{i, \Phi} \sin \Phi \\
q_{i}= & \sum_{k=5}^{8} \mu_{i k} N_{k} \cos \theta_{k}+\sum_{k=5}^{8} N_{i, \theta_{k}} \cos \theta_{k}+\sum_{\Phi^{*}} \epsilon_{\Phi^{*}} N_{i, \Phi^{*}} \cos \Phi^{*} \\
& +\sum_{\Phi} \epsilon_{\Phi} N_{i, \Phi} \cos \Phi \\
p_{i}= & \sum_{k=5}^{8} \mu_{i k} N_{k} \sin \theta_{k}+\sum_{k=5}^{8} N_{i, \theta_{k}} \sin \theta_{k}+\sum_{\Phi^{*}} N_{i, \Phi^{*}} \sin \Phi^{*} \\
& +\sum_{\Phi} N_{i, \Phi} \sin \Phi
\end{aligned}
$$

In these expressions, argument $\Phi^{*}$ corresponds to the case when all the $r_{j}$ of formula 1 are equal to zero, that is to say to the long period terms.

In $2, \varepsilon_{\Phi}$ has been defined by :

$$
\varepsilon_{\Phi}=\operatorname{sign}\left(\sum_{j=5}^{8} r_{j}+\sum_{j=5}^{8} l_{j}+\sum_{j=5}^{8} m_{j}\right)
$$

Matrices $\left(\lambda_{i k}\right)$ and $\left(\mu_{i k}\right)$ represent the matrices of the eigenvectors of the Lagrange Laplace solution.

Let us introduce the frequencies $g_{j}$ and $s_{j}$ and the phases $\beta_{j}$ and $\delta_{j}$ :

$$
\psi_{j}=g_{j} t+\beta_{j} \quad \theta_{j}=s_{j} t+\delta_{j} .
$$

The frequencies $g_{j}$ and $s_{j}$ are determined at each iteration when the Lagrange equations are integrated. Phases $\beta_{j}$ and $\delta_{j}$ and the quantities $M_{k}$ and $N_{k}$ of the formula 2 constitute the integration constants of the system of variables $k, h, q$, $p$. The other integration constants are $\lambda_{i 0}$ and $n_{i 0}$. The mean value $a_{i 0}$ of the semi major axis is obtained by : $n_{i 0}^{2} a_{i 0}^{3}=f\left(1+m_{i}\right)$ where $m_{i}$ is the mass of the planet $i$ in solar mass unit. 
TABLE I

Amplitude of the long period terms in the longitudes of Jupiter and Saturn. The unit is the arcsecond.

\begin{tabular}{lrrr}
\hline Argument & Period & $\bar{B}_{\Phi *, J}$ & $\widetilde{B}_{\Phi *, S}$ \\
\hline$\psi_{5}-\psi_{6}$ & 54069 & -5078 & 12487 \\
$2 \psi_{5}-2 \psi_{6}$ & 27034 & -326 & 800 \\
$3 \psi_{5}-3 \psi_{6}$ & 18023 & -13 & 32 \\
$2 \psi_{6}-2 \theta_{6}$ & 11993 & 25 & -61 \\
$\theta_{5}-\theta_{B}$ & $4977 \tau$ & 19 & -46 \\
$\psi_{5}+\psi_{6}-2 \theta_{6}$ & 15411 & 10 & -26 \\
$\psi_{5}-3 \psi_{6}+2 \theta_{6}$ & 9816 & -5 & 11 \\
\hline
\end{tabular}

\section{Study of the Sun-Jupiter-Saturn System}

The method used for the construction of the right hand members and for the integration of the equations was set up on the occasion of the study of the simplified system : Sun-Jupiter-Saturn. The construction of the right hand members of the Lagrange equations is made by handling Fourier series according to the methods developed by (Chapront et al, 1974).

The Lagrange equations are expressed in closed form in function of the true longitudes (Chapront et al, 1975). The method used for the integration of the variables $k, h, q, p$ is of the Krylov-Bogolioubov type, and is the one developed in (Bretagnon, 1990).

\subsection{Long Period terms in the Semi Major Axes and in the Longitudes}

We know, from the Poisson theorem, that there are no secular terms at the first and second order of the masses in the semi major axes. On the contrary these terms come up from the third order (see Duriez, 1978). When a general theory is dealt with, these terms appear in the form of long period terms of the third order before integration (Bretagnon and Simon, 1990). Their frequency being proportional to the planetary masses, they give, after integration, second order terms in the semi major axes and, by the 3rd Kepler law, terms of the first order with respect to the masses in the longitudes. These periodic terms have a very large amplitude. The amplitude of the highest term is 4 times greater than that of the great inequality $2 \lambda_{5}-5 \lambda_{6}$ of a 900 year period. We give the most important ones in table I, as well as their period in years.

\subsection{Determination of the Fundamental Frequencies}

The good convergence of the long period terms of the semi major axis and the longitude required many iterations.

Throughout these iterations the fundamental frequencies were determined very precisely. We found : 


$$
\begin{aligned}
& g_{5}=4.02683^{\prime \prime} \pm 810^{-5^{\prime \prime}} \quad s_{5}=0 . \\
& g_{6}=27.99622^{\prime \prime} \pm 13 \quad 10^{-5^{\prime \prime}} \quad s_{6}=-26.03608^{\prime \prime} \pm 8 \quad 10^{-5^{\prime \prime}}
\end{aligned}
$$

The uncertainties were evaluated from the results of the last iterations. They are confirmed by comparison of the solution to a numerical integration of the SunJupiter-Saturn system over 600000 years. On that time span, the greatest discrepancies between the general theory and the numerical integration do not exceed $5^{\prime \prime}$ for the longitudes, $10^{-5}$ for the $k$ and $h$ variables, $210^{-6}$ for the $q$ and $p$ variables.

The simplified problem which has been dealt with in this paragraph is obviously too far from the real problem to be used for the precise determination of the real frequencies from the values given in 5 .

\section{Study of the Sun-Outer Planets System}

We recently undertook an extension of this work to the system formed by the Sun and the 4 outer planets. One of the consequences is a very important increase of the size of the Fourier series representing the solutions.

The arguments $\Phi$ of the formula 1 are indeed linear combinations, no longer with 6 components, but with 12 : 4 mean longitudes, $4 \psi$ arguments and $4 \theta$ arguments. The Fourier series before integration are computed in arrays able to contain $2^{17}=131072$ terms, the solutions keeping only several thousand terms. For the level of precision which is now used, a complete iteration requires 1 hour computation on a CRAY Y-MP.

\subsection{Long Period terms in the Mean Longitudes}

Just as in the preceding study, we meet long period terms of large amplitude in the mean longitudes.

We give the amplitudes and periods of the most important terms of the longitudes for Jupiter and Saturn in table II, and for Uranus and Neptune in table III.

Just as for Jupiter and Saturn, the long period terms of Uranus and Neptune have a very large amplitude. For comparison, let us remind that the most important short period term of 4200 years period has an amplitude of $3000^{\prime \prime}$ in the longitude of Uranus and 2000" in that of Neptune.

The convergence is very slow all along the iterations and the amplitudes of tables II and III have now uncertainties of several arcseconds and even of many times ten arcseconds for the argument $\psi_{5}-\psi_{6}-\theta_{6}+\theta_{7}$ of period 2 million years in the longitudes of Jupiter and Saturn.

In the longitudes of the four outer planets can be found terms of large amplitude and greater period than in those of tables II and III (Milani, private communication). They deal with semi major axis terms of very low amplitude before integration. These terms are not yet obtained at the present state of precision of our computation. 


\section{TABLE II}

Long period terms of the study of the 4 outer planets in the longitudes of Jupiter and Saturn. The unit is the arcsecond.

\begin{tabular}{lrrr}
\hline Argument & Period & $\bar{B}_{\Phi^{*}, J}$ & $\bar{B}_{\Phi^{*}, S}$ \\
\hline$\psi_{5}-\psi_{6}$ & 54019 & -4675 & 11579 \\
$\psi_{5}-\psi_{7}$ & 1119981 & 1594 & -2675 \\
$\psi_{5}-\psi_{6}-\theta_{6}+\theta_{7}$ & 1987620 & 1000 & -2400 \\
$\psi_{6}-\psi_{7}$ & 51533 & 341 & -825 \\
$2 \psi_{5}-2 \psi_{6}$ & 27009 & -272 & 668 \\
\hline
\end{tabular}

TABLE III

Amplitude of the long period terms in the longitudes of Uranus and Neptune. The unit is the arcsecond.

\begin{tabular}{lrrr}
\hline Argument & Period & $\bar{B}_{\Phi^{*}, U}$ & $\bar{B}_{\Phi^{*}, N}$ \\
\hline$\psi_{5}-\psi_{7}$ & 1119981 & -20583 & 9996 \\
$\psi_{7}-\psi_{8}$ & 536444 & $-32 \tau$ & 218 \\
$\psi_{5}-\psi_{6}$ & 54019 & -262 & 13 \\
$\psi_{5}-\psi_{8}$ & 362713 & 215 & -206 \\
$\theta_{7}-\theta_{8}$ & 562479 & 156 & -44 \\
$\psi_{6}-\psi_{7}$ & 51533 & -111 & 22 \\
\hline
\end{tabular}

\subsection{Fundamental Frequencies}

The value of the fundamental frequencies is determined at every iteration with a still very slow convergence. We have estimated the uncertainty from the results of the last five iterations. The system which has been studied, that of the Sun and of the outer planets is very close to the real problem. We can give an estimate of the real frequencies using the contribution of the inner planets $\Delta g_{i}$ and $\Delta s_{i}$ to the Lagrange Laplace solution given in (Bretagnon, 1990).

We have

$$
\begin{array}{ll}
\Delta g_{5}=0.0116^{\prime \prime} & \Delta s_{5}=0 \\
\Delta g_{6}=0.0052^{\prime \prime} & \Delta s_{6}=-0.0082^{\prime \prime} \\
\Delta g_{7}=0.0010^{\prime \prime} & \Delta s_{7}=0.0003^{\prime \prime} \\
\Delta g_{8}=-0.0001^{\prime \prime} & \Delta s_{8}=0.0004^{\prime \prime}
\end{array}
$$

We give in table IV the frequencies thus obtained, as well as the results of Applegate et al (ADGSW, 1986), of Nobili et al (NMC, 1989) and Laskar (L 90, 1990).

\section{Conclusion}

The frequencies $g_{6}$ and $g_{7}$ that we have obtained still hold an important uncertainty. The other frequencies are a significant result. Throughout the coming iterations, the increase of precision in the computation should enable us to determine the 
TABLE IV

Fundamental frequencies associated to the onter planets. The unit is the arcsecond per year.

\begin{tabular}{lcrrr}
\hline & this work & $A D G S W$ & $N M C$ & \multicolumn{1}{c}{$L 90$} \\
\hline$g_{5}$ & $4.25 i 6 \pm 0.0005$ & 4.2422 & 4.25749 & 4.2488 \\
$g_{6}$ & $28.226 \pm 0.020$ & 28.2318 & 28.24553 & 28.2207 \\
$g_{7}$ & $3.092 \pm 0.010$ & 3.0905 & 3.08676 & 3.0895 \\
$g_{8}$ & $0.6694 \pm 0.0040$ & 0.6733 & 0.67255 & 0.6671 \\
$s_{5}$ & 0 & 0 & 0 & 0 \\
$s_{6}$ & $-26.345 \tau \pm 0.0025$ & -26.3324 & -26.34496 & -26.3302 \\
$s_{7}$ & $-2.9956 \pm 0.0005$ & -2.9948 & -2.99266 & -3.0056 \\
$s_{8}$ & $-0.6919 \pm 0.0002$ & -0.6923 & -0.69251 & -0.6919 \\
\hline
\end{tabular}

fundamental frequencies to an accuracy included between $10^{-4}$ and $10^{-3}$ arcsecond per year.

\section{Acknowledgements}

The computations presented in this paper were executed on a CRAY Y-MP belonging to the service Informatique et Mathématiques Appliquées of Electricité de France. We would like to thank the $I M A$ staff for giving us the use of this computer whose powerful resources were necessary for the realization of our work. We particularly express our gratitude to Madame Morin for her efficient help and precious advice in the adaptation and exploitation of our programs. We also deeply thank Madame Raoult for the precious help she gave us in this work.

\section{References}

Applegate, J.H., Douglas, M.R., Gursel, Y., Sussman, G.J., Wisdom, J.: 1986, Astron. J. $92(1), 176$.

Bretagnon, P.: 1974, Astron. Astrophys. 30, 141.

Bretagnon, P.: 1982, Astron. Astrophys. 114, 278.

Bretagnon, P.: 1984, in Milankovitch and Climate, Part I, eds. A.L. Berger et al., Reidel, p.41

Bretagnon, P.: 1990, Astron. Astrophys. 231, 561.

Bretagnon, P., Simon, J.L.: 1990, Astron. Astrophys. 239, 387.

Chapront, J., Chapront, M., Simon, J.L. : 1974, Astron. Astrophys. 31, 151.

Chapront, J., Bretagnon, P., Mehl, M.: 1975, Celes. Mech. 11, 379.

Duriez, L. : 1978, Astron. Astrophys. 68, 199.

Duriez, L.: 1979, Approche d'une Théorie Générale Planétaire en variables elliptiques héliocentriques, Thèse, Lille.

Kinoshita, H., Nakai, H.: 1984, Celes. Mech. 34, 203.

Laskar, J.: 1988, Astron. Astrophys. 198, 341.

Laskar, J. : 1990, Icarus 88, 266.

Milani, A., Nobili, A.M., Carpino, M.: 1987, Astron. Astrophys. 172, 265.

Nobili, A.M., Milani, A., Carpino, M.: 1989, Astron. Astrophys. $210,313$.

Quinn, T.R., Tremaine, S., Duncan, M.: 1991, Astron. J. 101 (6), 2287. 